\title{
A Versatile Memristor Circuit Emulator for Nanoscale Thin-film TiO2 Using Discrete-electronic Components
}

\author{
Van Ha Nguyena, Jakyeong Lee, Ala'aDdin Al Shidaifat, Jae Keun Song*, Hanjung Song ${ }^{\mathrm{b}}$ \\ Inje University, Department of Nanosience and Engineering, "Department of Physical Theraphy \\ 197 Inje-ro, Gimhae, Republic of Korea \\ anguyenha@oasis.inje.ac.kr; bhjsong@inje.ac.kr
}

\section{Extended Abstract}

The successful fabrication of a real nanoscale thin-film $\mathrm{TiO}_{2}$ memristor from Hewlett Packard (HP) Laboratories has greatly re-energized the interests of researcher in memristor and its future promising applications, especially in nanoscale era because of the limitations in CMOS technology [1]. Many works have started to utilized memristor in diverse nanoscale areas; especially non-volatile random access memory (ReRAM) and neuronmophic synaptic networks [2]. However, the solid memristor devices are not expected to be available in the near future because of the technical difficulties involved in the nanoscale fabrications. Therefore, a replacement which behaves like a real memristor device is urgently needed to allow researchers to study the practical application of memristors. In this study, we present a memristor emulator circuit which can correctly mimic the behaviour of the real nanoscale $\mathrm{TiO}_{2}$ thin-film memristor device from $\mathrm{HP}$ Labs. Additionally, the proposed emulator can also show various i-v hysteresis characteristics as observed at different dimensions of real memristor devices. The proposed emulator is a flux-controlled memristor emulator which originally reported in [3] based on a thirdorder polynomial function of flux-charge $\left(q=\alpha \phi+\beta \phi^{3}\right)$. The emulator circuit is constructed using off-the-shelf electronic devices with two operational amplifiers $\mathrm{U} 1$ and $\mathrm{U} 2$; two multipliers $\mathrm{U} 3$ and $\mathrm{U} 4$; one capacitor $\mathrm{C}$ and six resistors $\mathrm{R}, \mathrm{R}_{1}, \mathrm{R}_{2}$, $\mathrm{R}_{\alpha}, \mathrm{R}_{\beta 1}$, and $\mathrm{R}_{\beta 2}$. The success of constructing a controllable memristor emulator of $\mathrm{HP}^{\mathrm{TiO}}{ }_{2}$ memristor was confirmed by both of numerical simulation and laboratory experiment. The numerical analysis of the proposed scheme is firstly performed with an equivalent mathematical modelling in Matlab. The value of $\alpha$ and $\beta$ in the nonlinear flux-charge function were chosen for identifying the model parameters of electronics elements in the emulator in order to observe the typical i-v hysteresis loop of the real memristor. Different $\mathrm{i}-\mathrm{v}$ shape of the emulator circuit was obtained by changing coefficients $\alpha$ and $\beta$ in the nonlinear cubic polynomial. The emulator circuit was finally implemented on the prototype print circuit board (PCB) for the experimental observation of the memristive behaviour as well as the controllability using simple electronics devices such as AD633 multipliers; HA17741 amplifiers. Three potentiometers were used for $\mathbf{R}_{\alpha}, \mathrm{R}_{\beta 1}$, and $\mathrm{R}_{\beta 2}$ to control the memristive behavior of the proposed memristor emulator. Measurements of the controllable PCB-type memristor emulator were performed using oscilloscopes for the observation of its characteristics. All of the gained results from simulation and measurements were highly matched with the real thin-film $\mathrm{TiO}_{2}$ memristor presented in [1]; thus, the proposed memristor emulator is recommended as the foundation for understanding and design different emulators for nanoscale $\mathrm{TiO}_{2}$ thin-film memristors at the Laboratory level to engineers and scholars who cannot access memristor fabrication technology but want to studies memristor-based related.

\section{Acknowledgements}

This research was supported by Korea Electric Power Corporation through Korea Electrical Engineering \& Science Research Institute (R15XA03-66). This research was also supported by the Basic Science Research Program through the National Research Foundation of Korea(NRF) funded by the Ministry of Education, Science and Technology (NRF2015R1D1A1A01057495).

\section{References}

[1] D. B. Strukov, G.S Snider, D. R. Stewart and R.S. Williams, "The missing memristor found," Nature, vol. 453, pp. 8083, 2008. 
[2] P. Ontobel, W. Robinett, P. Kuekes, D. Stewart, I. Straznicky, and R. Williams, "Writing to and reading from a nano-scale crossbar memory based on memristors," Nanotechnology, vol. 20, no. 42, pp. 425204, 2009.

[3] B. Muthuswamy: "Implementing memristor based chaotic circuits," Int. J. Bifurcat. Chaos, vol. 20, no. 5, pp. 13351350, 2010. 